\title{
Low-Cost Multimodal Physiological Telemonitoring System through Internet of Things
}

\author{
Kadek Heri Sanjaya ${ }^{\text {a, * }}$, Asep Nugroho a ${ }^{\text {Latif Rozaqi }}{ }^{\text {a }}$, Yukhi Mustaqim Kusuma \\ Sya'Bana $^{\text {a }}$, Rizqi Andry Ardiansyah a ${ }^{\text {, Artha Ivonita Simbolon }}{ }^{\text {b }}$, Ulfah Nadiya ${ }^{\text {b }}$, \\ Dalna Nikita Ramdhani ${ }^{\mathrm{c}}$, Muhammad Akbar Maulana ${ }^{\mathrm{c}}$, Achmad \\ Fachturrohman $^{\mathrm{c}}$, Vyndi Myllazari $^{\mathrm{c}}$, Bhetri Sonia Yolandari ${ }^{\mathrm{c}}$, Lolita Agastya $^{\mathrm{c}}$ \\ ${ }^{a}$ Research Centre for Electrical Power and Mechatronics \\ Indonesian Institute of Sciences \\ Kampus LIPI, Jalan Sangkuriang \\ Bandung, Indonesia \\ ${ }^{b}$ Technical Implementation Unit for Instrumentation Development \\ Indonesian Institute of Sciences \\ Kampus LIPI, Jalan Sangkuriang \\ Bandung, Indonesia \\ ${ }^{c}$ Department of Biomedical Engineering \\ Institut Teknologi Sepuluh Nopember (ITS) \\ Kampus ITS Sukolilo \\ Surabaya, Indonesia
}

\begin{abstract}
The objective of this study is to develop and test a patient telemonitoring system. This study was encouraged by the high number of health workers fatalities in Indonesia due to physical contact without proper protection. Based on the symptoms of COVID-19 it consists of electrocardiogram (ECG) sensors, body temperature sensors, respiratory rate sensors, and pulse oximeter. The physiological data were captured by the sensors and collected by a microcontroller then it sends the data to a cloud system so that health workers can access the data. The experiments were performed to test both the offline and online protocol to compare data sent via a direct connection and data sent via Wi-Fi. In the offline testing, there were several limitations observed such as the low sampling frequency of the ECG signals that reduce the fidelity of the signals. Such problems were also observed on respiratory rate data. Furthermore, the system is also very prone to subjects' movement-related noise. The measurements of peripheral oxygen saturation $\left(\mathrm{SpO}_{2}\right)$ and body temperature, on the other hand, have been detected the slight change up to $0.1 \%$ and $0.5^{\circ} \mathrm{C}$ respectively. In the online testing, the data transmission to the cloud is sent per 30 seconds so that morphologically the ECG signal data are not representative. The system requires a lot of improvements and future study should be directed to improve signals acquisition and processing while maintaining the concept of low-cost. Design improvement should also include a better attachment design to the human body as well as greater data transmission for the online system.
\end{abstract}

Keywords: telemedicine, COVID-19, electrocardiography, oxygen saturation, respiratory, body temperature, web application.

\section{INTRODUCTION}

The concept of the fourth industrial revolution (or industry 4.0/I4.0) has been introduced by a group of German scientists tasked with the formulation of future high-tech strategy [1]. The concept proposes greater automation in industrial practices using the Internet of Things (IoT) and smart sensors that can analyze and diagnose problems without human intervention [2]. The cyber-physical systems of I4.0 combine hardware, software, and biology. While I4.0 has been growing in popularity worldwide, Japan, on the other hand, introduced Society 5.0 (S5.0) which implements cyberphysical systems not only to solve industrial issues but to answer social problems holistically without putting aside

\footnotetext{
* Corresponding Author.

Email: kade001@lipi.go.id

Received: May 07, 2021 ; Revised: June 23, 2021

Accepted: August 07, 2021 ; Published: August 31, 2021

Open access under CC-BY-NC-SA

(C) 2021 PPET - LIPI
}

the importance of economic advancement in a humancentered society [3]. Both Germany and Japan have problems with the aging population as such both I4.0 and S5.0 are directed to deal with the issue [4].

The application of IoT in healthcare provides several future possibilities [5][6]. IoT enables the greater connection between all people and things and greater sharing of medical data. Automation will reduce the burden for analyzing information and physical works so that the elder population will remain productive. The use of IoT augmented preventive health examination and robot-supported caregiving will lengthen life expectancy and reduce the social cost [7]. The implementation of S5.0, in the end, is expected to provide goods and services equally for all the population through the fusion of cyberspace and physical space.

Since early 2020, the world has been facing a global pandemic due to the SARS-Cov-2 virus. The virus was firstly identified from patients in Wuhan. It would become a global pandemic in a relatively short period of time because it can be transmitted rapidly from human to 
human. It is identified as Coronavirus Disease 2019 (COVID-19). In Indonesia, officially in March 2020, two patients were tested positive and become the first patients that suffer COVID-19. In a few months, the total cases of COVID-19 have been growing exponentially, that by early 2021 Indonesia has become the country with the highest number of cases in Southeast Asia. To make things worse, the number of COVID-19-related death among health workers in Indonesia is also among the highest in the world [8]. It is very crucial to protect the doctors since Indonesia has the second-lowest density of doctors among ASEAN countries [9].

The above-mentioned problem encouraged the development of a telemonitoring system to reduce physical contact between the health workers and suspect patients of COVID-19 through distant monitoring of physiological signals. The objective of this study was to develop and test the prototype of the telemonitoring system. The system requirements are low cost, easy to use with minimal intervention, and portable. The system was based on off-the-shelf instruments to make it affordable and easy to produce. In previous studies, telemonitoring system on COVID-19 patients was based on either self-report through SMS [10], and a happy hypoxia detection method using Max 30100 sensor [11]. Another study published before the pandemic developed a low-cost instrument to measure heart rate, blood oxygen saturation, and body temperature [12]. However, despite of more variables measured, the latter was not developed with the intention to reduce physical contact between health workers and the patients. Furthermore, the system did not record ECG data which may reveal more information on heart disorders. As such, the proposed telemonitoring system will be designed according to the consideration on COVID-19 pathological condition by combining sensors based on the most important symptoms of the disease. We also tested the prototype to evaluate its performance. This paper is divided into several parts. In section II, it presents the design process of the proposed telemonitoring system. The experiment procedure and results for testing the prototype are described in section III. Section IV discusses the prototype testing results. Finally, section $\mathrm{V}$ will conclude the several findings. Because the device is developed by using low-cost electronic components, this paper also gives several suggestions and guidance to improve reliability in the next design.

\section{The Proposed Telemonitoring System}

\section{A. Physiological Variables for the Proposed Telemonitoring System Design}

Indonesian Ministry of Health has issued guidelines to manage the pandemic which include information on the disease incubation period and its symptoms [13]. COVID-19 infection can cause mild, moderate, or severe symptoms. The main clinical symptoms that appear are fever (body temperature $>38^{\circ} \mathrm{C}$ ), cough, and difficulty in breathing. In addition, it can be accompanied by severe shortness of breath, fatigue, muscle pain (myalgia), gastrointestinal symptoms such as diarrhea, and other respiratory symptoms. Half of the patients developed shortness of breath within one week.

The heart is a great indicator of health [14]. Patients with COVID-19 have been reported to develop various types of heart disorders [15][16]. While the disorders are not solely caused by COVID-19 infection and more likely due to pre-infection systemic illness, it is widely understood that patients with a history of heart conditions and poor metabolic conditions have a greater risk to get more severe COVID-19 [17]. Infection by the virus and fever increases the heart rate and therefore the work of the heart. Instability of the blood pressure by a sudden spike or drop stress the heart and increase the oxygen demand that may lead to heart damage. In patients with pre-infection unhealthy hearts, cardiac arrests and arrhythmias have been reported to occur.

Another physiological condition suffered by COVID-19 patients is the decrease of oxygenation level both in the blood (hypoxemia) [18] and tissue (hypoxia) [19][20]. The condition of low oxygen is not always accompanied by short breath or dyspnea so that the patients seem to be clinically well. It is usually detected by using a pulse oximeter which measures peripheral oxygen saturation $\left(\mathrm{SpO}_{2}\right)$. Pulse oximeter estimates the oxygen percentage in the hemoglobin. Late detection of happy hypoxemic conditions may lead to fatality.

\section{B. Proposed System Development of Telemonitoring System}

Based on the measurements of the physiological variables in the patients, the telemonitoring system includes several physiological data acquisition systems such as heart rate monitoring sensors, body temperature sensors, respiratory rate sensors, and pulse oximeter. The overall system of low-cost telemonitoring based on IoT is shown in Figure 1. The data parameters from a suspect patient of COVID-19 were captured and collected by ESP8266 then it will ask for an HTTP request through ThingSpeak (as a broker) and health workers can access the data via the dashboard in the cloud system.

Heart rate sensors use the low-cost AD8232 sensor (Sparkfun Electronics, USA). The AD8232 sensor is a signal conditioning block capable of extracting, filtering, and amplifying very noisy biopotential signals from the body. This sensor gets this biopotential input signal

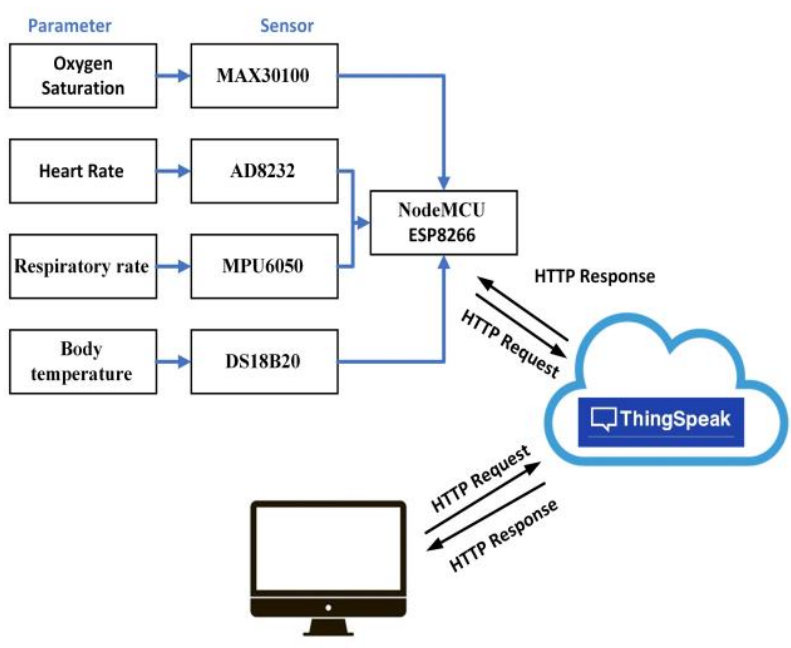

Figure 1. Proposed Low-cost Telemonitoring based on IoT. 
through electrodes that are placed on certain parts of the body based on Einthoven's triangular bipolar lead theory. This sensor is based on the 3 lead ECG technique, which uses a yellow electrode with a positive pole, a red electrode with a negative pole, and a green electrode as ground with an analog signal reading output. The electrode gets bioelectric signals based on the principle of contact between metal ions and the corresponding metal to produce an electric potential called the electrode potential. The electrode potential is generated due to the difference in the rate at which ions enter and leave the metal.

The AD8232 has employed a two-pole high-pass filter for eliminating motion artifacts and the electrode half-cell potential. By coupling, this filter and instrument amplifier is allowing to get large gain and high-pass filtering in a single stage, thereby saving space and cost. The required resistor and capacitor that puts high pass filter (pin HPSENSE) can be represented by (1).

$$
f_{c}=\frac{1}{2 \pi R C}
$$

Where $f_{c}$ is cut off frequency to block dc signal. The quality of the electrocardiogram (ECG) signal heavily depends on a low-impedance conductive path from the patient's body to the monitoring device. The results of the patient's physiology may not representative when any disruption occurred between the body and the monitoring device. The lead-off detection mechanism will notify the user when the electrodes are not connected properly.

The pulse oximetry included in the telemonitoring system is MAX30100 (Maxim Integrated Inc.). This sensor can monitor heart rate signals and the level of oxygen in the blood. This sensor consists of 2 LEDs and a photodetector. The MAX30102 operates at voltages between $1.8 \mathrm{~V}$ to $3.3 \mathrm{~V}$. This sensor uses I2C-based communication with the microcontroller. This sensor can also be activated and deactivated via software. This sensor has a programmable sample rate with the current in the LED to save power. The sensor is a reflectivebased sensor, which measures the heart rate when the index finger or wrist (as in this study) is brought near the red LED on the sensor. The LED will emit a light signal that can penetrate the capillaries in the skin. In the process of pumping blood by the heart that flows blood to the arteries, there is a change in the intensity of light. This change will be detected by the photodiode. The analog signal generated by these changes will be transmitted to the microcontroller where the signal will be further processed.

This sensor works by using the nature of hemoglobin which can absorb light and natural pulses of blood flow in the arteries to measure oxygen levels in the body. A device called a probe has a light source, light detector, and microprocessor that can compare and calculate the difference between oxygen-rich and oxygen-deficient hemoglobin. Hemoglobin that is richer in oxygen absorbs more infrared light, whereas hemoglobin which has no oxygen absorbs red light. The microprocessor on the probe calculates the difference in oxygen levels and converts this information into a digital value. This value is then estimated to determine the amount of oxygen carried by the blood. Relative light absorption measurements are made several times every second. The measurements are then processed by a microprocessor to provide a new result every $0.5-1$ second. The MAX30100 has a programmable analog to digital converter (ADC) up to 16-bit resolution. The MAX30100 sampling rate relies on ADC resolution and how long the period of pulse width is showed in Table 1.

MPU6050 sensors (TDK Electronics, Japan) are used for measuring respiratory rate and body temperature. It can read tilt angles based on data from the accelerometer sensor and the gyroscope sensor. The data line used on this sensor is the $\mathrm{I} 2 \mathrm{C}$ data line and can run on a power supply voltage of $3-5 \mathrm{~V}$. This sensor is very accurate with internal hardware facilities 16-bit ADC for each channel. This sensor will capture the $\mathrm{X}, \mathrm{Y}$, and $\mathrm{Z}$ axes channel values at the same time. This respiratory rate measurement method is based on changes in thoracic volume and body movements whereas body temperature is measured through on-chip temperature sensor output digitized by analog to digital converter (ADC). During inhalation and exhalation, there are periodic changes in volume in the thoracic and abdominal region with movements on the surface. These changes can be detected by inertia motion units (IMU/accelerometers and gyroscopes), imaging devices, and various sensors based on electromagnetic, piezoresistive, piezoelectric, and optical mechanisms. These constitute most of the methods that directly measure the Respiratory Rate. Accelerometers and gyroscopes can be used to capture signals that are modulated by respiration when positioned directly over the diaphragm. The position and orientation of the accelerometer and gyroscope are changed by the expansion and contraction of the chest wall during respiration. Accelerometers and triaxial gyroscopes provide three measurements of linear or angular acceleration in three orthogonal directions. The component required for the analysis is the direction of acceleration due to gravity in the accelerometer coordinate system, which is modulated by respiration.

One of the main symptoms of COVID-19 is fever, so the DS18B20 digital thermometer is operated as a body temperature monitoring. The device gives 9-bit to12-bit Celsius temperature measurements, and it can be completed by alarm function with nonvolatile userprogrammable upper and lower trigger points. The data stream sends through one wire protocol communication. There are 2 modes powering the device i.e., the parasitepowered and with an external power supply. In the parasite-powered mode, DS18B20 can derive power

TABLE 1

$\mathrm{SPO}_{2}$ MODE SETTING

\begin{tabular}{|c|c|c|c|c|}
\hline \multirow{2}{*}{$\begin{array}{c}\text { Sample } \\
\text { (Per second) }\end{array}$} & \multicolumn{4}{|c|}{ Pulse Width $(\boldsymbol{\mu s})$} \\
\cline { 2 - 5 } & $\mathbf{2 0 0}$ & $\mathbf{4 0 0}$ & $\mathbf{8 0 0}$ & $\mathbf{1 6 0 0}$ \\
\hline 50 & o & o & o & o \\
\hline 100 & o & o & o & o \\
\hline 167 & o & o & o & x \\
\hline 200 & o & o & o & x \\
\hline 400 & o & o & x & X \\
\hline 600 & o & x & x & x \\
\hline 800 & o & x & x & x \\
\hline 1000 & o & x & x & x \\
\hline ADC Res (bit) & 13 & 14 & 15 & 16 \\
\hline
\end{tabular}


directly from the data line and eliminating the need for an external power supply.

As showed in Table 2, in general, the choice of lowcost components to develop the telemonitoring system resulted in competitive cost compared to similar commercial products. We selected the products that were close with the proposed system from the market price. Considering the prototype is not yet at mass production stage, we may assume that the final product in the future has potential to be relatively cheaper than other products.

\section{Prototype Testing AND Results ANALYSIS}

As the main priority of the designed system is for this stage of development is affordability, it consists of low-cost sensors and a microcontroller. The testing of low-cost telemonitoring is shown in Figure 2. There are 3 subjects who participated in the testing. All of them were healthy young adult males aged 19-22 years taken from the university students who were involved in the development of the system. The biodata of subjects are provided in Table 3 . With regard to body mass index (BMI), the three subjects are varied. Subject 1 is moderate thinness, subject 2 is normal, and subject 3 is obese. All the subjects gave their consensual agreement to participate in the experiment. To collect data on the subject, conditioning was carried out on the subject so that the results of data retrieval are in accordance with the desired results and minimize the presence of unreadable data due to the subject's position when data collection is not suitable. After all tested instruments are installed on the subject's body in accordance with the position of the sensor that will record the subject's data, it is

TABLE 2

COST COMPARISON WITH OTHER SYSTEMS

\begin{tabular}{|c|c|c|}
\hline Items & Specifications & Cost \\
\hline $\begin{array}{l}\text { Coros Apex } \\
\text { Health and Fitness } \\
\text { Smart Watch }\end{array}$ & $\begin{array}{l}\text { GPS sensor, continuous } \\
\text { optical heart rate } \\
\text { monitor, accelerometer, } \\
\text { compass, body } \\
\text { temperature sensor }\end{array}$ & USD 529-600 \\
\hline $\begin{array}{l}\text { Whoop Strap } 3.0 \\
\text { Health and Fitness }\end{array}$ & $\begin{array}{l}\text { Continuous optical heart } \\
\text { rate monitor, oxygen } \\
\text { saturation } \\
\begin{array}{l}\text { Accelerometer, } \\
\text { compass, }\end{array} \\
\text { temperature body } \\
\text { lifestyle habits tracking, } \\
\text { intelligent rensor, } \\
\text { monitoring application, } \\
\text { support } \\
\text { networking }\end{array}$ & $\begin{array}{l}\text { USD } 30 / \text { month } \\
\text { (subscription } \\
\text { base with device } \\
\text { is free rent) }\end{array}$ \\
\hline $\begin{array}{ll}\text { Philips } & \text { Biosensor } \\
\text { BX100 } & \end{array}$ & $\begin{array}{l}\text { Respiration rate, heart } \\
\text { rate, ECG, oxygen } \\
\text { saturation, posture and } \\
\text { activity tracking, health } \\
\text { monitoring app for } \\
\text { clinician }\end{array}$ & $\begin{array}{l}\text { USD } 100-160 \\
\text { for device, USD } \\
40-60 / \text { month } \\
\text { (subscription } \\
\text { base) }\end{array}$ \\
\hline Kardia Alive Cor & $\begin{array}{l}\text { ECG monitoring system, } \\
\text { emergency alert for } \\
\text { clinician. }\end{array}$ & $\begin{array}{l}\text { USD } 139 \text { for } \\
\text { device, USD } \\
9.99 / \text { month } \\
\text { (subscription } \\
\text { base). }\end{array}$ \\
\hline Proposed device & $\begin{array}{lrr}\text { Heart rate } & \text { sensor, } \\
\text { oxygen } & \text { saturation } \\
\text { sensor, } & \text { temperature } \\
\text { sensor, support cloud } \\
\text { networking }\end{array}$ & $\begin{array}{l}\$ 215 \text { for device } \\
\text { (prototype) } \\
\text { No subscription }\end{array}$ \\
\hline
\end{tabular}

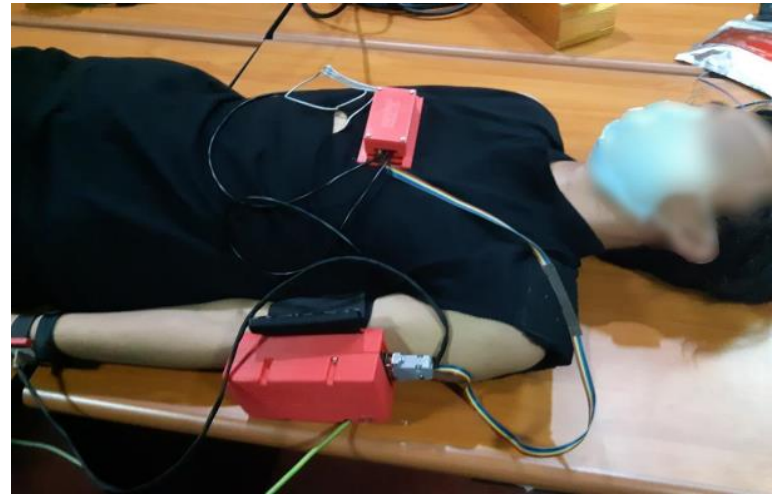

Figure 2. Testing Low-cost Telemonitoring based on IoT.

TABLE 3

BIODATA OF SUBJECTS

\begin{tabular}{|l|l|l|l|l|}
\hline & Age & Height & Weight & BMI \\
\hline Subject 1 & 21 year & $173 \mathrm{~cm}$ & $49 \mathrm{~kg}$ & 16.4 \\
\hline Subject 2 & 19 year & $163 \mathrm{~cm}$ & $65 \mathrm{~kg}$ & 24.5 \\
\hline Subject 3 & 22 year & $170 \mathrm{~cm}$ & $100 \mathrm{~kg}$ & 34.6 \\
\hline
\end{tabular}

recommended that the subject lay supine in a relaxed state and do not make much movement to minimize unreadable data while recording. Subjects are advised to remain in position during data collection.

\section{A. Offline Experiment}

Making offline programming for this multi-modal monitoring system was intended to anticipate conditions where the device cannot connect to $\mathrm{Wi}-\mathrm{Fi}$ as well as to compare data sent via a direct connection and data sent via Wi-Fi. In addition, we also compared data from the device with the data recorded by the modules of Biopac MP 160 (Biopac Systems, USA). The Biopac System is a modular physiological data acquisition system widely used for laboratory-based life science research [21]. The offline method was chosen because the results of the reading data from the wearable device can be stored and viewed again. Thus, it is easy to compare the results of the ECG and Respiratory Rate of the tools that have been made. Although the microcontroller used is the ESP8266 Wi-Fi module, this application can still be used for offline reading as long as the program uploaded to the microcontroller has not used the Wi-Fi library. The design of the device for this monitoring system has undergone several adjustments due to various things so that the application made is also adjusted to the device. In the early stages of research, all sensors were connected to the same microcontroller. After testing the system, a problem was found where the baud rate between the temperature sensor and other sensors had different values. This caused data transmission to experience delays. The baud rate value for the ECG AD8232 module and the MAX30100 pulse oximeter sensor is at 115200 while the D10B20S temperature sensor is at 9600 . Therefore, the experiment was carried out using two microcontrollers. However, after using this method the results are unreadable by the laptop. What happened was that the laptop only managed to read one microcontroller and the data received were random and not sequential. This causes the data received to be at different times by the laptop. 
After conducting a literature study, the best solution was obtained, namely by using one module ESP8266. The references suggested that ESP8266 module has the lowest cost [22]-[24]. The module is also easy to use and consume very low power [23][24]. A previous study also reported that it was applicable for distant recording of ECG data [25]. In our study, the use of ESP8266 was successful in sending data in real-time, but later another problem was found. In a system that uses one microcontroller, data readings from the four sensors will be read and sent simultaneously. The ESP8266 is programmed using Arduino and reads as COM6 on the laptop. However, the ECG data experiences lagging when using one microcontroller. ECG data can still be read, but many are skipped so that the accuracy is reduced. But the results for other sensors are better with one microcontroller so that it was decided to continue to use one microcontroller.

In the first stage, the heart rate and electrocardiogram (ECG) signals were collected through the AD8232 sensor module then they were compared to the commercial biomedical testing device Biopac MP 160. The ECG signals recorded by Biopac MP 160 systems at a $2000 \mathrm{~Hz}$ sampling rate whereas the ECG signals recorded by the developed systems were sampled at only $9 \mathrm{~Hz}$. The difference in sampling rate due to the much lower resolution of the AD8232, especially in multimodal sensors recording, generated different waveforms. The ECG signals recorded by the Biopac Systems were smooth and show the QRS complex well as depicted in Figure 3(a). On the contrary, the low sampling rate of the designed telemonitoring systems resulted in a square and tooth type of waveforms. It is shown in Figure 3(b).

The unexpected finding shows that using a pooling structure for sampling ADC from AD8232 module is not a good idea. Although the clock frequency of ESP32 is high enough, the pooling structure causes a bottleneck if two or more tasks are running at the same time. Ideally, a critical task such as sampling ADC should be done in high priority interrupt and managed by the real-time operating system (RTOS).

As shown in Figure 4, the results of the CrossCorrelation analysis of the ECG signals from the instrument tested with the ECG signals from the RSPEC Biopac MP 160 module recorded from subject 1 indicate the amount of data is 180 . If the analysis is carried out, the midpoint of the cross-correlation results is the point where the time delay is at a value of 0 then if it moves to the left, the time delay will be negative, and if it moves right, the time delay will be positive. There is a global maximum peak that occurs in the 138 and 158 delay sequences, which shows the highest correlation level of the ECG signals from the instrument being tested with the ECG signals from RSPEC. Meanwhile, the minimum peak value occurs in the delay sequence value of 69 .

The results of the Cross-Correlation analysis of the ECG signal from the instrument tested with the ECG signal from the RSPEC Biopac MP 160 module recorded from subject 2 show that the data fluctuates with global maximum peaks occurring in sequence delay 77 and global minimum peaks occurring at sequence delay 36 .

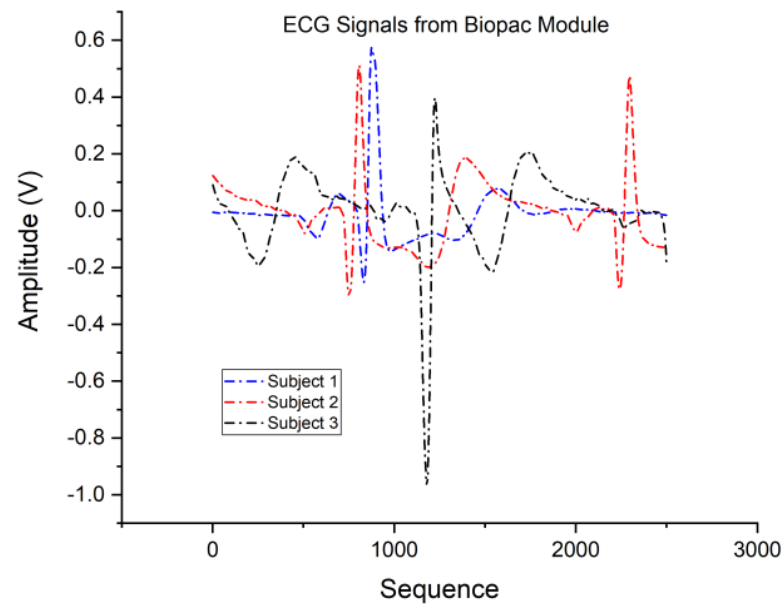

(a)

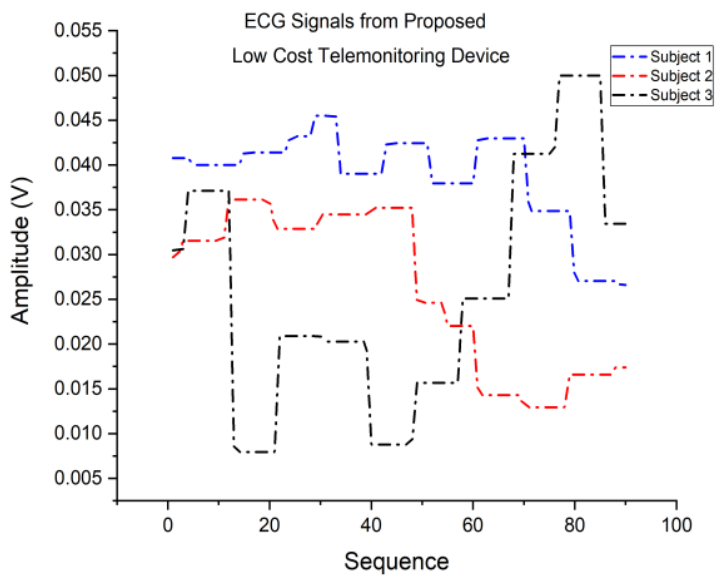

(b)

Figure 3. (a) ECG Signals from The Three Subjects Recorded by Biopac MP 160 RSPEC Module (b) By Low-cost Medical Telemonitoring Device.

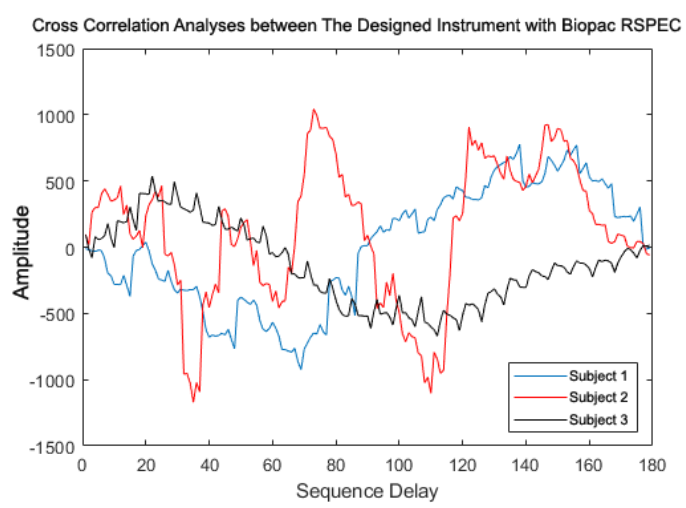

Figure 4. The Results of Cross Correlation Analyses between The ECG Signals Recorded by The Designed Instrument and Those Recorded by The Biopac MP 160 RSPEC Module.

These results indicate that the association between the ECG signal from the instrument being tested and the ECG signal from RSPEC has several high correlation points (3 maximum peaks) and several low correlation points ( 2 minimum peaks).

Moreover, the subject 3 results show that there is noise in the entire data range starting from sequence delay 0 to 180 . This is because subject 3 is very obese with thick subcutaneous fat that obstruct biosignals 
transmission to the surface electrode. Observations on the results of the analysis show that the global maximum peak is in sequence delay 22 while the global minimum peak is in sequence delay 113 . From the experiments that have been carried out on the three subjects, the results of the cross-correlation graph of the proposed instrument ECG signal with the ECG from the RSPEC Biopac MP 160 module do not represent the ECG signal in general because the raw data on the system from the proposed tool is different from the sampling rate with the ECG raw data from the RSPEC module.

Like the ECG signals, the respiratory rate data also show an obvious difference between the data collected using BIOPAC MP 160 systems and those collected by the designed telemonitoring systems. The difference is quite huge, and it is shown in Figures 5(a) and 5(b). The visual difference of the graph is furthermore supported by the results of cross-correlation analysis where the values deviate far from zero which indicate great time delay and waveform dissimilarities between the data collected by the two instruments. However, both subjects 1 and 2 have negative time delay whereas subject 3 has positive time delay the cross-correlation analysis in subject 1 shows that the resulting maximum amplitude value was $0 \mathrm{mv}$ and the minimum amplitude value was $2 \mathrm{mv}$. The maximum correlation value occurs at points 0 and 180, while the minimum correlation value occurs at point 90 . In subject 2 , the results of the cross-correlation of the respiratory rate signal from the designed instrument with a respiratory rate signal from the RSPEC

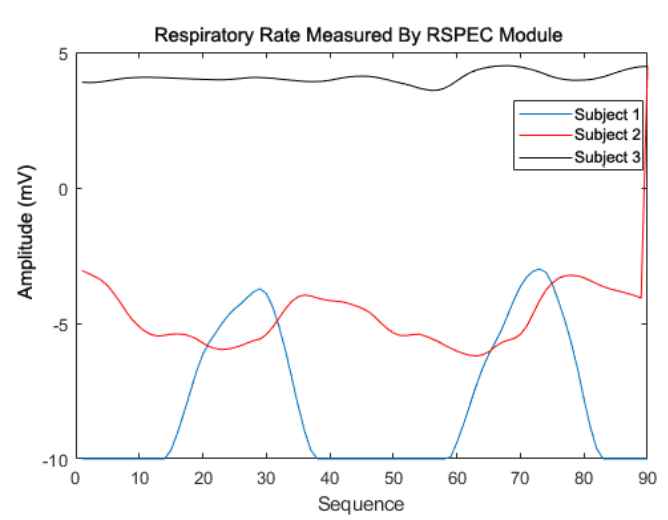

(a)

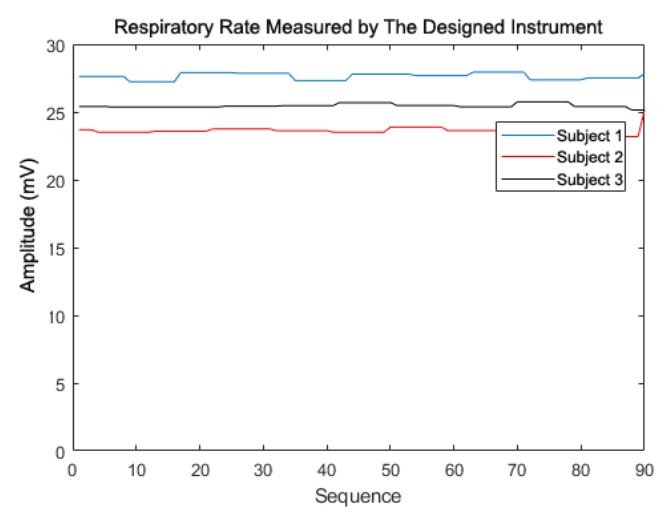

(b)

Figure 5. (a) Respiratory Rate Signals from The Three Subjects Recorded by Biopac MP 160 RSPEC Module (b) By The Designed Instrument.
Biopac MP 160 module showed a maximum amplitude value of $9000 \mathrm{mv}$ and a minimum amplitude value of 0 mv. The maximum correlation value occurs at point 90 . Meanwhile, the minimum correlation value occurs at points 0 and 180 . The results of cross-correlation analyses on signals from subjects 1 and 2 showed similarity in waveforms despite differences in value. In the measurement of subject 3 , the results of the crosscorrelation of the respiratory rate signal from the instrument tested with the respiratory rate signal from the RSPEC Biopac MP 160 module showed the maximum amplitude value produced was $0 \mathrm{mv}$ and the minimum amplitude value was $-10000 \mathrm{mv}$. The maximum correlation value occurs at points 0 and 180 , while the minimum correlation value occurs at point 90 . The results in subject 3 showed a difference with the results of both subjects 1 and 2 in value and waveforms. It is depicted in Figure 6. We speculate the difference was occurred because of the obesity of subject 3 that may affect the fidelity of the data.

Normally, the level of oxygen in the blood needed by the body to function optimally is $95-100 \%$, when measured with a pulse oximeter. Experiments in this study were carried out by placing a pulse oximeter sensor MAX30102 on the subject's wrist. It can detect the change of oxygen level up to $0.1 \%$. As depicted in Figure 7 , the measurement of $\mathrm{SpO}_{2}$ in subject 1, subject 2, and subject 3 who are healthy subjects, so that before the measurement, it is expected that the $\mathrm{SpO}_{2}$ measurement results from the sensor are above the normal threshold. In

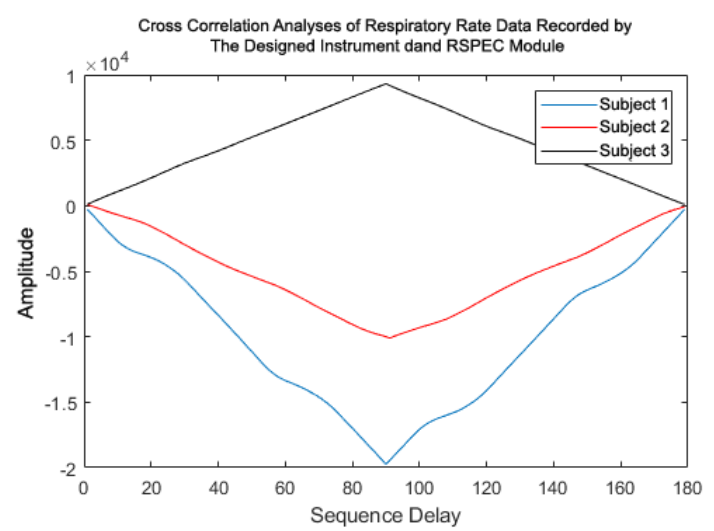

Figure 6. Cross Correlation Analyses of Respiratory Rate Data Recorded by The Designed Instrument and Biopac MP 160 RSPEC Module on The Three Subjects.

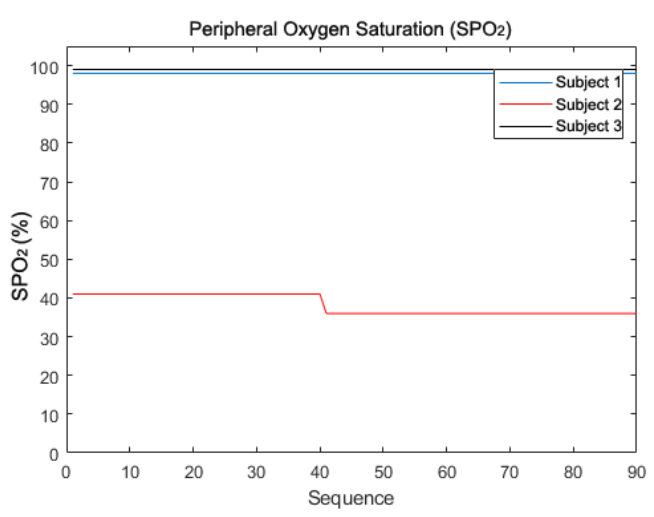

Figure 7. Oxygen Saturation $\left(\mathrm{SpO}_{2}\right)$ Data from The Three Subjects as Measured by The Designed Instrument. 
subject 1 , the $\mathrm{SpO}_{2}$ level was observed to be stable at a percentage of $98 \%$ oxygen in the blood. From the measurement results in subject 2 , the percentage of oxygen levels in the blood was unstable and very low between $41 \%$ and $36 \%$. This may be due to the fact that subject 2 data collection, the installation, and placement of the sensor were not optimal in terms of both the placement and attachment, and maybe subject 2 made movements that caused noise when recording data. Therefore, the value obtained is far below the expected value before the experiment. Finally, from the measurement results in subject 3 , the percentage of oxygen levels in the blood was also stable at $99 \%$.

Normal human body temperature measured from the surface of the body at certain anatomical points is generally between $36.5^{\circ}-37.2^{\circ}$ Celsius. Normal body temperature can change throughout the day because daily activities can also affect body temperature. Usually, the temperature will rise to $0.6^{\circ}$ Celsius throughout the day depending on the activities that each person has done. Experiments were carried out by placing the DS18B20 temperature sensor on the right armpit of the subject.

As illustrated in Figure 8, the body temperature testing on subjects 1,2 , and 3 was carried out for approximately 3 minutes. The result of body temperature measurement by the DS18B20 temperature sensor in subject 1 is stable at $35^{\circ}$ Celsius. Then in the results of measuring the body temperature from the right armpit in subject 2 , the temperature was also stable at $36^{\circ}$ Celsius. However, the results of the measurement by DS18B20 temperature sensor on subject 3 slightly fluctuated due to errors that occurred caused by subject movement. The movement moved the sensor so that it was detached from the surface of the subject's armpit skin which caused disturbance to data recording. Based on datasheet, the DS18B20 has $\pm 0.5^{\circ} \mathrm{C}$ of accuracy so it can be acceptable in medical standard for temperature measurement.

\section{B. Online Monitoring}

The initial appearance of the website online monitoring can be seen in Figure 9. On the home page of the website, there are several features that can be accessed by users. To be able to access the data that has been sent, users simply click the "Start Monitor" button. After that, the user will be directed to the Sign-Up or login page. For new users, they must wait for verification from the administrator before being able to $\log$ in and

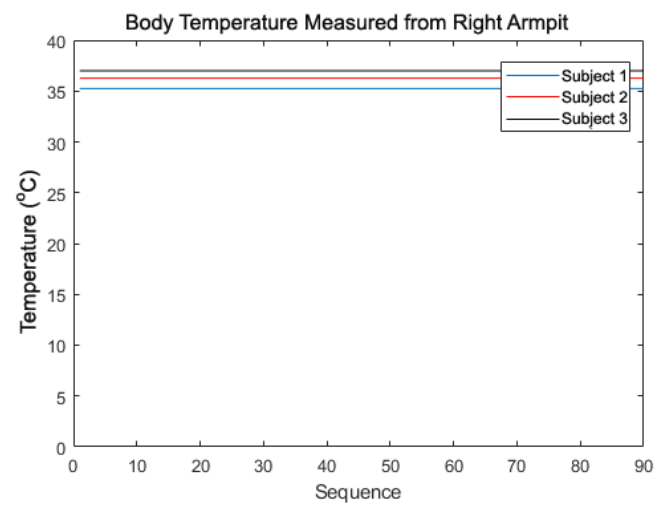

Figure 8. Body Temperature Data from The Right Armpit of The Three Subjects as Measured by The Designed Instrument.

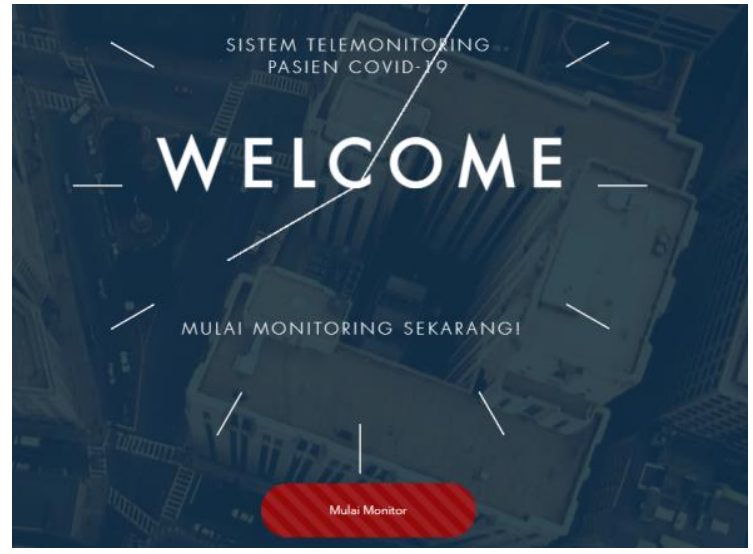

Figure 9. Log In Page of The Website for Online Monitoring in Bahasa Indonesia.

access the data that has been sent. Meanwhile, for verified users, the data from the patient who is currently using the device will be displayed. A verification procedure is necessary to keep patient medical records.

From the description, it is understood that the data displayed is in accordance with what was sent. However, the weakness of this system is that data transmission to the cloud is sent per 30 seconds so that the ECG signal data cannot be displayed according to the ECG morphology that should be. In addition, data reset cannot be done directly on the website page but must be done by administrators through Thingspeak so that when changing patients, it is necessary to inform the administrator first.

\section{DISCUSSION}

Heart rate, respiratory rate, $\mathrm{SpO}_{2}$, and body temperature are among the primary vital signs that are often used for assessing patients' conditions [26]. The early detection of heart disorders either through a stethoscope or ECG is very crucial to save lives [27]. With regard to ECG signals, the three most common variables measured in most studies are heart rate, arterial blood pressure, and local blood volume [28]. The minimum sampling rate of ECG signals recording is recommended to be no less than $500 \mathrm{~Hz}$ to be able to analyze various variables [29]. The proposed telemonitoring system, however, is only able to achieve a $9 \mathrm{~Hz}$ sampling rate so that the data cannot show any measurable variables of heart activities. ECG signals consist of various PQRST complex, where $\mathrm{P}$ wave represents atrial depolarization, QRS represents ventricular depolarization, and $\mathrm{T}$ wave represents ventricular repolarization [30]. COVID-19 has been reported to be associated with cardiac arrhythmia [15][16]. The various aspects of an arrhythmic heart can be analyzed through the PQRST complex assessment. As such, the capacity of the telemonitoring system to capture the representative morphology of ECG signals is very crucial and should be a priority for future improvement.

Shortness of breath is one obvious symptom of COVID-19 as the disease infects the lower respiratory tract. The importance of respiratory rate in detecting COVID-19 makes it one suggested variable for the telemonitoring system during self-isolation by using a wristband-attached photoplethysmography (PPG) sensor 
[31]. However, in this study, the respiratory rate measurement was measured from the thoracic volume change by the MPU6050 sensors. The sensors failed to record the respiratory rate. During the experiment, the wrist was the body part where the MAX30100 pulse oximeter sensor was placed and it was relatively more successful in recording the $\mathrm{SpO}_{2}$ data. MAX30100 sensor has been reported to have accuracy between $96-99 \%$ [12][11]. In further studies, it is necessary to investigate the best alternative of respiratory rate measurement methods and instruments such as accelerometer, PPG, etc.

Higher body temperature has been associated with a greater risk of mortality among COVID-19 patients, where there is a greater risk of mortality every $0.5^{\circ} \mathrm{C}$ body temperature increase above the $37^{\circ} \mathrm{C}$ thresholds [32]. In this study, the DS18B20 body temperature sensor placed on the armpit successfully recorded the body temperature data to measure the change of $0.5^{\circ} \mathrm{C}$. The relatively slow change of temperature means the low sampling rate of the system has no significant effect.

The slow data transmission for the online monitoring system may be due to both the system capacity and network connection. Other than improving the system, especially several strategies could be tested. The use of a dedicated network and server apart from the public network as used in the experiment to increase data transmission speed is probably a good alternative. Another method is probably a buffering system that collects and transmits data continually within several periods.

In general, the overall measurement performance of the proposed low-cost medical telemonitoring device has many limitations and still far below the standard of medical equipment. The issues include the low accuracy of ECG and respiratory rate detection, because of low sampling frequency and body movement-related noise. Several suggestions for the next development such as improving ADC sampling rate, using high priority interrupt for data sampling, handling multiple tasks should be using a real-time operating system (RTOS), increasing noise to signal ratio (SNR) using an adaptive digital filter, and create better standard operation procedure when collecting data. A dedicated interface and information system design such as in a previous study [33] will optimize the user experience. Two concepts that should be kept in future studies are low-cost and off-the-shelf components, as those concepts are very appropriate for telemedicine in a developing country like Indonesia [34]. For future study, a comparison of the proposed system performance with other commercial products is also necessary.

\section{CONCLUSION}

The telemonitoring system is necessary to reduce the contact between health takers and COVID-19 patients in hope of decreasing the fatalities of health takers of COVID-19 cases in Indonesia. The low-cost concept was introduced to ease the manufacturing process and more widespread acceptance. However, the use of low-cost components in the designed telemonitoring system resulted in several limitations such as the low sampling frequency of the ECG signals that reduce the fidelity of the signals. The ECG signals are not representative when compared to signals generated by Biopac Systems MP 160 ECG module. Such problems were also observed on respiratory rate data. Furthermore, the system is also very prone to movement-related noise so that subjects' movement during the experiment should be limited. The measurements of $\mathrm{SpO}_{2}$ and body temperature, on the other hand, have been able to detect the change in oxygen saturation and body temperature. In the online system, data transmission is also very slow that creating a representative signal with higher sampling frequency is impossible. Future study should be directed to improve signals acquisition and processing while maintaining the concept of low-cost. Design improvement of the telemonitoring system design, when attached to the human body is also necessary in order to allow more freely movement of the patients. Improvement on the data transmission for the online system and comparison with other products are also necessary.

\section{ACKNOWLEDGMENT}

This study was funded by National Priority Research on COVID-19 of the Deputyship of Engineering Sciences (PN Kedeputian IPT 2020), Indonesian Institute of Sciences (LIPI).

\section{REFERENCES}

[1] Z. Li, Y. Wang, and K. S. Wang, "Intelligent predictive maintenance for fault diagnosis and prognosis in machine centers: Industry 4.0 scenario," Adv. Manuf., vol. 5, no. 4, pp. 377-387, 2017 .

[2] K. H. Sanjaya, L. Rozaqi, G. Y. W. Simanungkalit, A. N. Iksan, and M. Y. Yuriis, "Design of pressure measurement instrument for smart footwear," Internetworking Indonesia J., vol. 12, no. 1, pp. 3-8, 2020

[3] "Society 5.0," $2018 . \quad$ [Online]. Available: https://www8.cao.go.jp/cstp/english/society5_0/index.html (accessed May 5, 2021).

[4] Keidanren, "Healthcare in society 5.0," Japan Business Federation, Japan, 2018

[5] G. Ioppolo, F. Vazquez, M. G. Hennerici, and E. Andrès, "Medicine 4.0: New technologies as tools for a society 5.0," J. Clin. Med., vol. 9, no. 7, Jul. 2020, Art no. 2198.

[6] G. Aceto, V. Persico, and A. Pescapé, "Industry 4.0 and health: Internet of things, big data, and cloud computing for healthcare 4.0," J. Ind. Inf. Integr., vol. 18, Jun. 2020, Art no. 100129.

[7] A. I. Simbolon, K. H. Sanjaya, and A. Widyotriatmo, "EMG patterns of healthy subjects with shoulder rehabilitation robot," in Proc. 2019 IEEE 4th Int. Conf. Technology, Informatics, Management, Engineering and Environment, Nov. 2019, pp. 6771.

[8] WHO Indonesia, "Coronavirus Disease 2019 (COVID-19) Situation Report," 2021. [Online]. Available: https://www.who.int/indonesia/news/novelcoronavirus/situation-reports

[9] C. Kanchanachitra et al., "Human resources for health in southeast Asia: Shortages, distributional challenges, and international trade in health services," Lancet, vol. 377, no. 9767, pp. 769-781, 2011.

[10] A. U. Morgan et al., "Remote monitoring of patients with Covid19: Design, implementation, and outcomes of the first 3,000 patients in COVID Watch," New England J. Medicine Catal., pp. 1-12, Jul. 2020

[11] N. N. Sari, M. N. Gani, R. A. M. Yusuf, and R. Firmando, "Telemedicine for silent hypoxia: Improving the reliability and accuracy of Max30100-based system," Indones. J. Electr. Eng. Comput. Sci., vol. 22, no. 3, pp. 1419-1426, Jun. 2021.

[12] E. A. Suprayitno, M. R. Marlianto, and M. I. Mauliana "Measurement device for detecting oxygen saturation in blood, heart rate, and temperature of human body," in J. Phys. Conf. Ser., 
vol. 1402, no. 3, Dec. 2019, Art no. 033110

[13] Pedoman Tatalaksana Covid-19 (Guidelines for the Management of COVID-19), Kemenkes RI (Indonesian Health Ministry), 2020.

[14] O. Heriana and A. M. Al Misbah, "Comparison of wavelet family performances in ECG signal denoising," Jurnal Elektronika dan Telekomunikasi, vol. 17, no. 1, pp. 1-6, Aug. 2017.

[15] A. N. Kochi, A. P. Tagliari, G. B. Forleo, G. M. Fassini, and C. Tondo, "Cardiac and arrhythmic complications in patients with COVID-19," J. Cardiovasc. Electrophysiol., vol. 31, no. 5, pp. 1003-1008, May 2020.

[16] A. Bhatla et al., "COVID-19 and cardiac arrhythmias," Hear. Rhythm, vol. 17, no. 9, pp. 1439-1444, Sep. 2020.

[17] D. K. L. Lewis. "COVID-19 and the heart: What have we learned." Harvard Health Publishing Harvard Medical School. https://www.health.harvard.edu/blog/covid-19-and-the-heartwhat-have-we-learned-2021010621603 (accessed May 03, 2021).

[18] M. J. Tobin, A. Jubran, and F. Laghi, "Misconceptions of pathophysiology of happy hypoxemia and implications for management of COVID-19," Respir. Res., vol. 21, Sep. 2020, Art. no. 249.

[19] A. González-Duarte and L. Norcliffe-Kaufmann, "Is 'happy hypoxia' in COVID-19 a disorder of autonomic interoception? A hypothesis," Clin. Auton. Res., vol. 30, no. 4, pp. 331-333, Jul. 2020.

[20] A. Widysanto et al., "Happy hypoxia in critical COVID-19 patient: A case report in Tangerang, Indonesia," Physiol. Rep., vol. 8, no. 20, pp. 1-5, Oct. 2020, Art. no. 14619.

[21] "ECG: Cardiology | Heart Sounds | Research | BIOPAC." https://www.biopac.com/application/ecg-cardiology/advancedfeature/heart-sounds/ (accessed May 24, 2016).

[22] D. R. P. Patnaikuni, "A Comparative study of Arduino, Raspberry Pi and ESP8266 as IoT development board," Int. J. Adv. Res. Comput. Sci., vol. 8, no. 5, pp. 2350-2352, Jun. 2017.

[23] J. Mesquita, D. Guimaraes, C. Pereira, F. Santos, and L. Almeida, "Assessing the ESP8266 WiFi module for the internet of things," in IEEE Int. Conf. Emerging Technologies and Factory Automation, Oct. 2018, pp. 784-791.

[24] R. K. Kodali and K. S. Mahesh, "A low cost implementation of MQTT using ESP8266," in Proc. 2016 2nd Int. Conf. Contemporary Computing and Informatics, 2016, pp. 404-408.
[25] A. Škraba, A. Kolozvari, D. Kofjac, R. Stojanovic, E. Semenkin, and V. Stanovov, "Prototype of group heart rate monitoring with ESP32," in Proc. 2019 8th Mediterranean Conf. Embedded Computing, Jun. 2019.

[26] G. Sun et al., "Vital-SCOPE: Design and evaluation of a smart vital sign monitor for simultaneous measurement of pulse rate, respiratory rate, and body temperature for patient monitoring," $J$. Sensors, vol. 2018, 2018, Art. no. 4371872.

[27] P. Oktivasari, R. Riandini, R. A. Fitri, and S. I. Malaon, "Active filter analysis on designing electronic stethoscope," Jurnal Elektronika dan Telekomunikasi, vol. 19, no. 2, pp. 51-56, Dec. 2019.

[28] J. P. J. Pinel, Biopsychology, Bahasa Ind. Yogyakarta: Pustaka Pelajar, 2015.

[29] J. J. Bailey et al., "Recommendations for standardization and specifications in automated electrocardiography: Bandwidth and digital signal processing. A report for health professionals by an ad hoc writing group of the committee on electrocardiography and cardiac electrophysiology of the council on clinical cardiology, American heart association," Circulation, vol. 81, no. 2, pp. 730739,1990

[30] H. Carter, "Cardiology- the ABC's of the PQRST," Bush Veterinary Neurology Service, 2018.

[31] D. J. Miller et al., "Analyzing changes in respiratory rate to predict the risk of COVID-19 infection," PLoS One, vol. 15, no. 12, Dec.2020, Art. no. 0243693.

[32] S. Tharakan, K. Nomoto, S. Miyashita, and K. Ishikawa, "Body temperature correlates with mortality in COVID-19 patients," Crit. Care, vol. 24, Jun. 2020, Art. no. 246.

[33] K. H. Sanjaya, L. Rozaqi, K. A. Damayanti, M. M. Laurentius, and Hamilaturrohmah, "Design of Smart Footwear Information System for Measuring Gait Parameters," in 2019 Int. Conf. Computer, Control, Informatics and its Applications: Emerging Trends in Big Data and Artificial Intelligence, 2019, pp. 76-81.

[34] E. D. Kurniawan, A. Adam, M. I. Salik, and P. L. Gareso, "Programmable Syringe Pump for Selective Micro Droplet Deposition," Jurnal Elektronika dan Telekomunikasi, vol. 19, no. 2, pp. 75-82, Dec. 2019. 